\title{
SPATIAL ANALYSIS OF BORDER CLOSURE INTERVENTION SCHEME IN CONFLICT-INDUCED DISPLACEMENT
}

\author{
Zahra Jafari \\ Department of Security and \\ Crime Science \\ University College London \\ London, United Kingdom \\ zahra.jafari.17@ucl.ac.uk
}

\author{
Toby Davies \\ Department of Security and \\ Crime Science \\ University College London \\ London, United Kingdom \\ toby.davies@ucl.ac.uk
}

\author{
Shane D. Johnson \\ Department of Security and \\ Crime Science \\ University College London \\ London, United Kingdom \\ shane.johnson@ucl.ac.uk
}

\begin{abstract}
Since 2011, Syria has experienced episodes of large-scale conflict and violence. Syrian Civil War has exposed the global society to increasingly flow of refugees who are forcibly displaced from their homes due to persecution, conflict, violence, or human rights violations. The inconsistent responses from humanitarian organisations and governments indicate lack of robust framework to assess and evaluate policy responses. In this article, we examine the effect of border control policies via a data-driven agent-based model of refugee flows from Syria. We implement policy scenarios and investigate the consequences in the flow pattern in terms of measuring the size of flows and the potential destinations. We demonstrate that the spatial configuration of borders intended to be closed directly influence the consequences of the corresponding closure policy.
\end{abstract}

\section{Author Keywords}

Agent-based model; migration; refugees; Syria; policy intervention; border closure;

\section{INTRODUCTION}

\section{INTRODUCTION}

The use of evidence to inform policy has become an increasing focus in many fields, in line with a general aim to promote more rigorous policy-making processes. One such field of application concerns migration, and in particular forced migration; that is, the forced displacement of individuals due to factors such as conflict or climate change. There are currently approximately 80 million $^{1}$ individuals globally who have been forcibly displaced from their homes, with many of these fleeing conflict; this comes as no surprise, given that approximately $12 \%$ of the world's population were living in active conflict zones as of 2016 (Charlson et al., 2019). Not

\footnotetext{
${ }^{1}$ UNHCR Global Trends
}

only are such large-scale movements dangerous for the individuals enduring such journeys, but the resulting flows present substantial social and economic pressures in destination countries. The rapid - and, at first sight, unpredictable - growth of these flows is a substantial challenge for both formal humanitarian efforts and ongoing accommodation.

The pressures described above give rise to a number of policy questions, and investigating the subsequent effect of potential intervention schemes is a crucial step in addressing these. Possible interventions include the provision of aid or the manipulation of economic influences (either in origin or destination locations), but also include the more explicit addition or removal of barriers (e.g. at border crossings). To understand the consequences of such policies requires us to develop an underlying understanding behind forced displacement; this, however, presents a substantial challenge. While both qualitative and quantitative insights are required, data in the field is scarce and hence quantitative analysis of forced displacement is subject to substantial uncertainty. This issue is particularly acute for refugee migration, due to its covert nature: many flows are likely to fall outside those recorded by administrative agencies.

As well as these data challenges, forced migration displays a number of characteristics which make the prediction of flows particularly difficult. The fact that migrants tend to follow the paths of previous migrants, for example - there is evidence that the presence of established networks of diaspora in potential destinations acts as an attractor (Massey et al., 1993) - means that systems of migration display path-dependence, whereby final outcomes are strongly influenced by the choices of early migrants (Simon, 2019). Furthermore, uncertainties and delays with respect to information flows are likely to cause non-linearities and feedback effects. Taken together, these properties suggest that forced migration displays the properties of a complex system.

Given the range of questions in need of quantitative answers, mathematical modelling has the potential to be of value in contributing to evidence-based policy making. As well as the ability to encode relevant behaviours, the analysis of such models can offer insight into likely outcomes in hypothetical circumstances, thereby acting as a test-bed for potential interventions. Such testing would, of course, be both impractical and unethical via traditional empirical approaches. Within modelling approaches, agent-based modelling [ABM] 
in particular is a natural approach in this context, given the combination of individualised decision-making and complexity outlined above. Furthermore, the ability to situate such models spatially, incorporating real-world data, is a distinct advantage of the computational approach, meaning that findings are both grounded in, and relatable to, the real-world context. Since the early rise of agent-based models, there have been several models of migration (Klabunde and Willekens, 2016; Frydenlund et al., 2019), and forced migration in particular has been the subject of recent studies (Kniveton et al., 2012; Suleimenova et al., 2017; Frydenlund et al., 2018). These models aim to quantify and explain the dynamics of migration by simulating the behaviours of migrants as well as the relevant environments and policies. Given the diverse range of ABMs in migration, as well as the limited capacity of each model to take many variables and procedures into account, each $\mathrm{ABM}$ serves a unique purpose in the geopolitical context of its own. Simon et al. introduced a data-driven ABM, focused on non-refugee migrants, to explore the effect of restrictive immigration policies on the migration patterns (Simon et al., 2018). Among the latest ABMs in the context of refugee migration, Suleimenova et al. estimate the final destination choices, given the number of refugees, based on limited variables such as distance and calculated speed (Suleimenova et al., 2017). Another recent work by Hebert et al. presents an agent-based model of movements from Syria. Although the model includes a range of variables as well as a suitable decision-making mechanism regarding the context of displacement, it leaves a gap for data calibration and policy exploration (Hébert et al., 2017).

Migration is a phenomenon that is subject to frequent policy shifts and spatial changes. Hence, exploring the effect of external and spatial interventions is vital to the lives of migrants as well as the policy-making process. In this work, we shed light on geographical policy interventions in the case of Syrian refugees. We explore policy scenarios through an agent-based model as a way to systematically capture the subsequent spatio-temporal changes.

Since the outbreak of Syrian civil war in 2011, the emergence of large refugee flows from Syria to Lebanon, Turkey, Iraq, Jordan, and countries across Mediterranean sea has exposed the global society and humanitarian organisations to many questions. Those questions also exist for the case of internally displaced people [IDP] moving between governorates within Syria. According to the United Nations High Commissioner for Refugees [UNHCR] refugee data, there are currently 6.2 million people displaced within Syria and 6.6 million Syrian refugees all across the globe, mostly residing in Turkey, Lebanon, Jordan, Germany and Iraq ${ }^{2}$. These movements are highly influenced by the geographical features of the origin and destination, such as distance, network of routes, and border conditions, as well as the environmental factors that vary across time and space, such as measure of conflict, camps and shelters, unemployment and food insecurity (Haas et al., 2019). Among these, border closures and tightly controlled land borders have imposed a number of restrictions on Syrians' decision to flee, which sometimes left them with only a few legal pathways to take refuge from conflict and poor livelihood

\footnotetext{
${ }^{2}$ https://www.unhcr.org/refugee-statistics/
}

3 . This often leads to populations becoming stranded near the borders, as well as surpluses of displaced people elsewhere, particularly as humanitarian camps are overwhelmed and suffer from insufficient food and hygiene resources (Crawley et al., 2017).

Humanitarian organisations and authorities have been experiencing inadequately prepared policy response and lack of plans for resource allocation for Syrian refugees in the midst of events and incidents (Carlson et al., 2018). Given the rise of migrants' death across Mediterranean sea, with 5,063 recorded deaths during $2016^{4}$, organisations and governments have been enquired about their immigration and border policies as well as the protection for refugees. Given the need for a systematic framework to evaluate policy scenarios, estimating the magnitude of flows at each point in the geographical space and its changes under various conditions is a key step. Mathematical modelling of such geographical system can contribute to fill said gaps since it investigates a range of scenarios and provides rigorous answers to the questions informing policies. These explorations, in tandem with empirical evidence, try to explain the changes in spatio-temporal patterns of IDP and refugee flows as a result of policy interventions.

In this work we briefly introduce an agent-based model, incorporating a spatial network of Syrian governorates and the neighboring countries, to simulate movements from and within Syria. After calibrating the model with empirical data, we discuss border closure scenarios and the ways in which it would influence spatial pattern of movements.

\section{AN AGENT-BASED MODEL OF REFUGEE FLOWS FROM SYRIA}

We use agent-based modelling as a tool to simulate conflictinduced movements of IDPs (within Syria) and refugees (outside Syria). The ABM simulates a system of agents, representing members of the Syrian population, who consider, at each step of the simulation, whether to stay in their home location or move elsewhere. Further, it simulates the destination choice of those who decide to move, determining the chosen destination for each agent. These decisions are based upon environmental and spatial variables which evolve over the course of time.

\section{The model environment}

The model environment takes the form of a simplified spatial representation of Syria and its neighbouring countries. The environment is represented as a set of discrete locations: the 14 governorates of Syria and its four immediate neighbours Lebanon, Turkey, Iraq and Jordan. These are arranged in a spatial network, in which each region is represented by a node and links represent the borders between them. The spatial network holds the geographical distances between nodes, calculated using Open Street Map. In addition, each node holds information about the intensity of conflict, number of camps, hospitals, border functionality and average wage. These variables are taken into account when agents make decisions with respect to whether to flee and where to travel.

\footnotetext{
${ }^{3}$ Closing Borders, Shifting Routes: Summary of Regional Migration Trends Middle East by Danish Refugee Council

${ }^{4}$ https://missingmigrants.iom.int
} 


\section{The agents}

The model is populated by agents representing Syrian individuals, each of which is located at one of the regions of the environment (i.e. one of the nodes). At the start of the simulation, agents are distributed across space in proportion to the population distribution within Syria. The number of agents depends on the population resolution: here this is set such that each agent represents 500 Syrian individuals. The population of agents therefore replicates the size and distribution of the Syrian population, with its size downscaled by a factor of 500 . Each agent is characterised by its location - that is, its current home - and income. Their experience and awareness at any point in time is determined by their location, since factors such as conflict intensity, average wage, camp availability and potential destinations vary across time and space.

The first stage of decision making involves the agents deciding whether to stay in their current home location or move elsewhere. This decision depends on: 1) the level of conflict in the area they live, measured in terms of the number of violent incidents during that month and the month before; 2) the number of camps available in the origin; and 3) the ratio of functioning and partially functioning hospitals. If this first stage results in the agent deciding to move, the second stage involves agents then choosing a destination to travel towards. This decision is also influenced by the current location of the agent: the decision depends on the closest potential destinations, the functionality of borders, the average wage and number of camps at potential destinations, and distance.

\section{Modelling decision making}

There are a number of theories which propose to explain how migrants of different kinds make decisions, taking into account their motivations and their reactions to environmental cues. These include aspiration-ability, bounded rationality, and rational choice theories (Klabunde and Willekens, 2016). Bounded rationality and heuristic decision-making theory explains heuristics as optimal processes that derive benefit from only a part of information (Gigerenzer and Gaissmaier, 2011). Heuristics save time and effort while allowing for heterogeneity and situation-based decisions in multifactorial decisionmaking.

In addition to these theoretical foundations, our model was also grounded in data derived from a large-scale survey of Syrian refugees residing in Lebanon in 2018 (Chu et al., 2019). This survey included both itemised questions and a focus group component, in which refugees discussed their experience of migration, including their motivations and decision-making processes.

In our survey data, out of 1750 respondents in Lebanon, more than half of them described their decision to leave Syria as a reaction to the life-threatening situation at home. The rest reported family reunion and economic situation as their main reason to leave. Such decisions are mostly taken at short notice as a result of a sudden incident. Many Syrian refugees described shelter, food availability and minimum income to afford food as factors guiding them towards their choice of destination. However, it was also clear that the stories narrated by migrants were not uniform, with each being distinct to the individual. Therefore, it came clear that the decisions do not follow the same rationality, while nevertheless making perfect sense within each individual's own rational frame. Since individuals, in situation such as these, cannot consider all factors and consequences, we gathered that often one or two cues shape decisions, whilst these cues differ across individuals. In the scope of this paper, the heterogeneity of the ABM is maintained through the probabilistic nature of the decisionmaking model, as well as the spatial distribution of the agents, such that agents from various origins decide differently due to their space-dependent exposure. However, agents from the same origin share similar behavioural regime that generates slightly different decisions as determined by the stochasticity embedded in the model.

According to the survey data, conflict plays the most important role in Syrians' decision to flee. Distance and economic opportunities as well as camp availability - camps as a shelter to access food, accommodation and hygiene facilities - are shaping the destination choice. However, there are restrictive policies such as routes and border closures as well as complex variables such as family ties that are diversifying migration decisions. Given the findings from survey data and the migration literature, we gathered that the decision process resembles a procedure as follows:

1. At each time $t$, the agent calculates both the utility of staying, $U_{s}(j, t)$, and the utility of leaving, $U_{l}(j, t)$, in their current location $j$. The former depends on hospital functionality and camp availability. The latter is a function of conflict during the current month and the previous month. The coefficients $a_{i}$ and $b_{i}$ are to be empirically calibrated.

$$
\begin{aligned}
& U_{s}(j, t)=a_{0} H(j, t)+a_{1} C(j, t) \\
& U_{l}(j, t)=b_{0} V(j, t)+b_{1} V(j, t-1)
\end{aligned}
$$

2. The probability of leaving is calculated based upon the utilities above and the agent chooses whether to leave or stay accordingly. The probability is modelled as a sigmoidal function of the difference between utilities, reflecting the likely presence of a 'tipping point' of relative attractiveness. The precise form of the function is determined by a parameter $d$, which we aim to calibrate empirically.

$$
p=\frac{U_{l}-U_{s}}{d+U_{l}-U_{s}}
$$

3. Once the agent decides to move, the agent probability to becomes either a refugee (crossing border), with probability $p_{r}$, or an IDP (moving internally), with probability $(1-$ $\left.p_{r}\right)$. This probability is calculated based upon the average functionality of their $k$ closest borders, with an additional tuning parameter $p_{1}$.

$$
p_{r}=p_{1}\left\langle b_{t}^{j}\right\rangle_{k n n}
$$

4. In case the agent decides to cross a border, they evaluate the attractiveness of $k$ closest borders and choose one based upon the corresponding attractiveness. The attractiveness depends on the distance between origin and potential destinations, reflecting the functional form of a classic 'gravity' model: $d_{i j}$ represents the distance between origin $i$ and 
destination $j$.

$$
P\left(X=x_{j}\right)=\frac{b_{t}^{j} e^{-\alpha_{1} d_{i j}}}{\sum_{k=0}^{k=m} b_{t}^{j} e^{-\alpha_{1} d_{i k}}}
$$

5. An internally displaced person evaluates attractiveness for each potential destination based upon the average wage and the number of camps in the destination, as well as the distance between origin and destination. The final destination $X$ is chosen probabilistically in proportion to these attractiveness values.

$$
P\left(X=x_{j}\right)=\frac{e^{-\left(\alpha_{1} d_{i j}-\alpha_{2} w_{j}(t)-\alpha_{3} C(j, t)\right)}}{\sum_{k=0}^{k=m} e^{-\left(\alpha_{1} d_{i k}-\alpha_{2} w_{k}(t)-\alpha_{3} C(k, t)\right)}}
$$

\section{Data}

The simulation covers the months from January 2016 to June 2019. Data from various sources is incorporated to the basic simulation as follows:

1. Insecurity is measured using the number of active conflict incidents in each governorate. This data was retrieved from Live Universal Awareness Map, an open data platform combining and mapping reports of conflict incidents. Potential duplicate incidents were removed and the monthly totals for each governorate were calculated.

2. The presence and functionality of healthcare facilities is measured using the number of functioning or partiallyfunctioning public hospitals within each governorate, based on data from the World Health Organization.

3. The number of IDP refugee camps within each governorate is based on data collated by the Humanitarian Information Unit (U.S. Department of State) in June 2015. Since only one snapshot of this variable is available, it is treated as time-invariant.

4. The average wage is calculated based on data from the World Food Programme's global food database.

5. Data regarding the functionality of border crossings is derived from various UNOCHA reports on border closures in Syria. Once there is at least one crossing point open that is recorded as an open border, hence border functionality is equal to 1 . If all crossing points are partially open we record the variable as 0.5 , and if they are all closed we assume the border functionality is 0 .

6. To validate and calibrate the model, we use the IDP population data reported by OCHA services; Humanitarian Response ${ }^{5}$. To complement the data, we count for refugees at the neighbouring countries by incorporating the Syrian refugees population data from $\mathrm{UNHCR}^{6}$.

\section{Calibration}

The scarcity of data in migration studies, particularly relating to refugee flows, has left many models and simulations of migration with limited options with respect to calibration.

\footnotetext{
${ }^{5}$ www.humanitarianresponse.info/

${ }^{6}$ https://data2.unhcr.org/en/situations/syria
}

Indeed, in many cases, calibration and validation of models is based on qualitative comparison between model outputs and data. In this work, we designed the decision mechanism in a way that was consistent with the data structure in order to allow for the systematic calibration of model parameters. The decision process as explained above consists of two separate stages: first, a decision to leave an origin, and second, the choice of destination. Accordingly, we implemented two separate calibration processes to first estimate the parameters in stage 1 which influence the flee decisions, and second, the destination choice parameters in stage 2 .

Data limitations limit the granularity with which calibration can be performed. For example, data concerning refugee flows - that is, flows to other countries - is limited to counts of aggregated arrivals, and the origins of migrants (i.e. their home governorates) are not known. For IDPs, on the other hand, origin-destination flow data is available via UNOCHA, as detailed above. The calibration procedure therefore seeks to fit model outputs to separate origin and destination flows to aggregated counts.

We used a Genetic Algorithm [GA] approach in order to calibrate the parameters in the decision process (Mohammadi et al., 2017). Although the calibration of ABM using GA has been done before (Heppenstall et al., 2007), a systematic empirical calibration in the simulation of refugee movements is a new step forward, since previous work stopped short of a full integration of data (Hébert et al., 2017). In the case described here, the calibration is driven purely by the data, with the result that the calibrated simulation generates patterns that replicate empirical migration data to a close (and quantifiable) extent. The correspondence between data and model outputs provides partial validation of the model, and acts as a foundation for further scenario explorations and experiments. Since the calibration contributes to a foundation for further policy explorations, it covers the entire time window offered by the datasets above.

The data incorporated in GA is the combination of IDP dataset and the UNHCR refugee dataset introduced above. The fitness function (i.e. the quantity that the GA seeks to minimise) measures the difference between the overall flow (inflow and outflow) at each month across all spatial nodes, from simulation and data. The calibrated parameters identified by this procedure are given in Tables 1 and 2.

Table 1. Flee simulation parameters derived from GA calibration

\begin{tabular}{cccccc}
\hline \hline Parameters & $a_{0}$ & $a_{1}$ & $b_{0}$ & $b_{1}$ & $d$ \\
\hline Estimated Values & 0.0153 & 0.8466 & 0.3929 & 0.2322 & 2.4328 \\
\hline
\end{tabular}

Table 2. Destination choice parameters derived from GA calibration

\begin{tabular}{ccccc}
\hline \hline Parameters & $p_{1}$ & $\alpha_{1}$ & $\alpha_{2}$ & $\alpha_{3}$ \\
\hline Estimated Values & 0.2429 & 0.8264 & 0.4196 & 0.8192 \\
\hline
\end{tabular}

\section{MODEL EXPERIMENTATION:}

Having been calibrated as described above, the model can be used to test the effectiveness of policy interventions by manipulating the conditions within the simulation. An example 
of such an intervention, which frequently arises in migrationrelated discourse, is the closure of borders. Border closures and hard entry visa restrictions are enforced by governments in order to stop flows of migrants into their countries, often in response to acute influxes (and influenced by political pressures). Such actions have acted as substantial constraints on the journeys of Syrians since the outbreak of civil war, with closures implemented both in nearby countries and further afield (e.g. in Europe).

The consequences of border closures are difficult to anticipate, in part due to the complexity of the migration decision process. In a spatial system, the tightening of border controls at one point may result in partial or entire flows being redirected towards other destinations; in other words, the flows are displaced elsewhere. The form of such displacement is not straightforward, however: individuals will have different relative preferences with respect to destinations, and the presence of social effects means that their choices may be dependent on those of others. Indeed, the removal of an option may mean that an individual no longer chooses to migrate at all, even if other destinations remain available.

The experiments below aim to deepen insights into the ways that border intervention schemes affect patterns of migration, and the consequential changes to flows elsewhere. After enforcing the closure of a border during a certain time window, we investigate the places expected to experience a surplus of displacement as well as the magnitude of change in the spatial pattern of displacement. We also compare the proportion of border crossings to internal displacements, and the distribution of number of journeys per head across space.

\section{Control experiment}

The aim of the control experiment is to establish a base case for further experiments, while also acting as a verification of the ABM and its calibration. The control experiment reflects the conditions that occurred in Syria between January 2016 and May 2019, and therefore is intended to replicate the patterns and behaviours observed in real-world data. The parameters are derived from the GA calibration as reported in Tables 1 and 2, and the datasets explained in the previous section provide input conditions consistent with empirical data. The simulation generates the flows of individuals leaving their homes and travelling either to other governorates in Syria or to neighbouring countries. Summing these for each governorate therefore gives the number of individuals fleeing from that origin.

The aggregate and disaggregated outcomes are broadly in agreement with the observed data, at both country level and governorate level. Figure 1 shows the total monthly flows originating from Syria for both simulation and data. Aside from the large spike at around month 22 in the empirical data - corresponding to missile strikes conducted by Israeli Air Force - the time series are in broad agreement. Given that empirical data cannot realistically be replicated exactly using a simplified model such as the one presented here, the fact that key trends are reproduced is an encouraging indication of the model's validity. The remaining variation may be explained by factors that are not included in the model (and for which data are limited) such as unemployment, poverty, and commu-

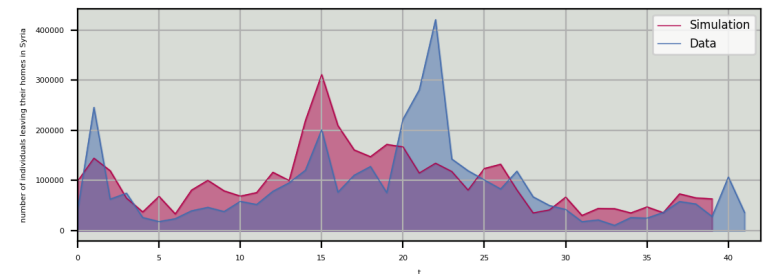

Figure 1. Country level size of monthly flows originating from Syria, from simulation and data. The simulation covers flows from Jan 2016 to May 2019, with simulation outputs rescaled to be comparable with empirical data.
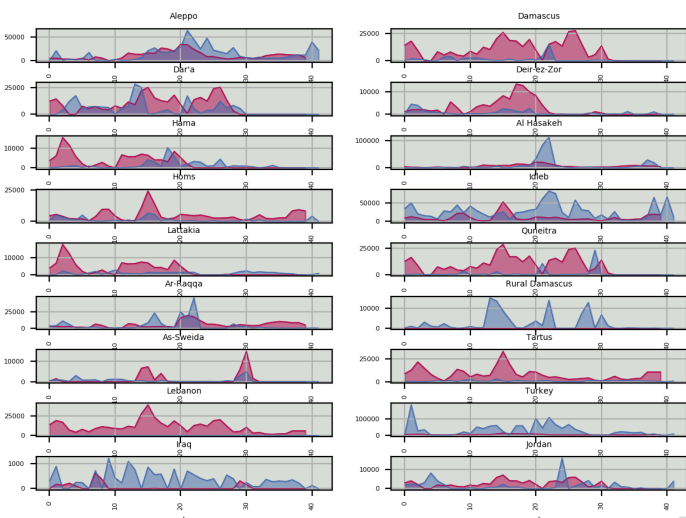

Figure 2. Governorate level monthly flows towards Syrian governorates and neighbouring countries, from simulation and data. The simulation covers flows from Jan 2016 to May 2019. Public data provided by UNHCR indicates zero official refugee registration records in Lebanon.

nication between groups.

The inflows towards governorates and neighbouring countries resulting from the destination choice model are also shown in Figure 2. The quality of agreement here is mixed: while key features such as peaks do coincide, there are mismatches in scale in some cases, particularly with respect to international crossings. Again, this discrepancy is likely to be due to factors not incorporated - such as kinship, food availability, accessibility and safety of roads - and, more importantly, the relatively coarse spatial resolution of the model in terms of population density and conflict incidents. While governorates are treated here as single entities, the exact locations of both agents and conflict incidents are likely to exert a substantial effect on spatial choice, particularly in large governorates: the effect of conflict is likely to be attenuated if it is occurring far away from an agent's location. It is worth noting that the UNHCR dataset, as the only accessible alternative, counts only the number of registered refugees in neighboring countries. Not only it does not include all refugees but also their entrance date does not necessarily correspond the registration date. Hence, the incorporated dataset does not represent the monthly number of border crossings. 
Closing Borders with Turkey

To simulate the intervention of border closure, we generate and incorporate an alternative time-series of border functionality in which the borders with Turkey are closed from November 2016 until September 2017. The parameters used in the simulation are the same as the control experiment. The aim of the experiment is to explore the changes in the flow pattern during the closure time window. These changes are reflected in agents' behaviours and decisions, and consequently in the generated flows. We study how the system adapts to the change made in the environment by analysing the generated time-series.

\section{Closing Borders with Iraq}

Each border plays a distinct role in the spatial network on which the model is situated. Specifically, each border plays a different role in the decisions of migrants, determined by its position relative to the populations of agents. This heterogeneity means that different border closures will lead to the emergence of distinct patterns, and that the effect of a border closure scheme will by shaped by the location of the border within the spatial system. The most important factors that characterise borders include the neighbour nodes in their proximity as well as the number of crossing points they maintain. In order to explore this heterogeneity, and the distinctive characteristics of the emergent patterns, we implement an additional border closure scenario. In the same spirit as the previous experiment, the borders with Iraq are shut between November 2016 and September 2017. The analysis is done to gain an understanding of how different spatial profile of borders influence the pattern of movements.

\section{RESULT}

\section{Closing Borders with Turkey}

The simulation is run given a synthetic border closure scenario in which Turkish borders are closed from November 2016 until September 2017. As illustrated in Figure 2, the simulation generates time series for each of the 18 possible destinations, reflecting the number of individuals travelling there at each month. In order to measure the discrepancy between the generated inflows from the control experiment and under the closure scenario, a difference ratio is defined and calculated. The discrepancy for each governorate $j$ at each month $t$ - defined as $D(j, t)$ - is measured as below, where $f(j, t)$ is the time-series generated under the control experiment and $f^{\prime}(j, t)$ is the time-series from the border closure experiment.

$$
D(j, t)=\frac{f^{\prime}(j, t)}{\sum_{j} f^{\prime}(j, t)}-\frac{f(j, t)}{\sum_{j} f(j, t)}
$$

Since the ABM is stochastic, both $f$ and $f^{\prime}$ can vary across runs of the experiment. Both are therefore calculated by averaging over 100 simulations, in order to account for potential noise in individual runs. The resulting measure $D(j, t)$, calculated using these averaged values, therefore measures the difference in the proportion of migrants travelling to $j$ between control and closure simulations.

As illustrated in Figure 3, after closing the borders with Turkey, the inflow towards Turkey vanishes as expected. This certainly influences the inflow towards other destinations, whether

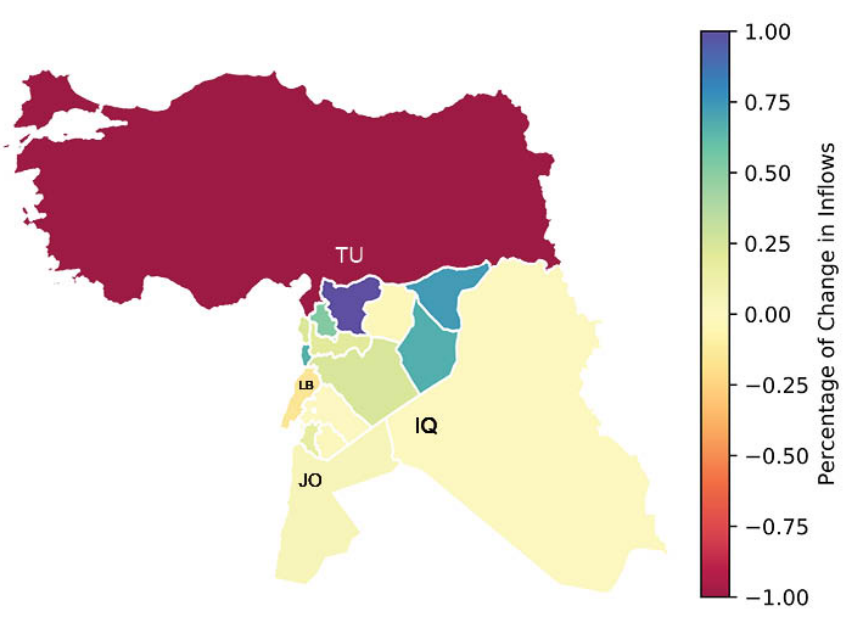

Figure 3. Change of inflow towards possible destinations as a result of Turkish border closure. Regions are coloured according to $D(j, t)$ - the difference between the outcomes from the control experiment and the Turkish border closure experiment - averaged over the 10 months of closure. Once the inflow towards Turkey dropped, Aleppo, Al-Hasakah and Deir ez Zur were the choice of many displaced persons.

across international borders or within the country. In general, the highest increases are seen for governorates in northern Syria: Aleppo, Al-Hasakah, and Deir ez Zur were the governorates which experienced the highest increases in their inflows during the ten month period of border closure (3). These increases include, on average, 1663, 1150, and 1038 more individuals at Aleppo, Al-Hasakah, and Deir ez Zur respectively. The primary displacement resulting from the closure of all borders with Turkey is therefore to IDP movement rather than legal border crossing; indeed, it results in a net decrease in international migration. To better understand the flow dynamics and the association between them, we examined the effects on inflows to alternative locations during the 10 months of intervention scheme in a pairwise sense. The correlogram in Figure 4 shows the association between displacements - that is, the correlation in $D(j, t)$ for pairs of destinations $j$ and $j^{\prime}$ - and shows a number of non-trivial effects. For example, the positive correlation between Hama and Jordan suggests that they are affected equivalently by displacement, whereas Damascus and Tartous experience opposite effects. Perhaps as notable are the low correlations in some cases: Iraq and Jordan show negligible correlation in their effects, despite being immediate neighbours.

\section{Closing Borders with Iraq}

Similar to the previous experiment, a set of simulations is run under the Iraqi border closure condition, with the same averaging technique applied to the simulation output. We compare this experiment to the base case experiment. Figure 5 illustrates the average discrepancy measure $D(j, t)$ defined in the previous section, reflecting the difference between the simulation output under the new border closure condition and the control experiment output. 


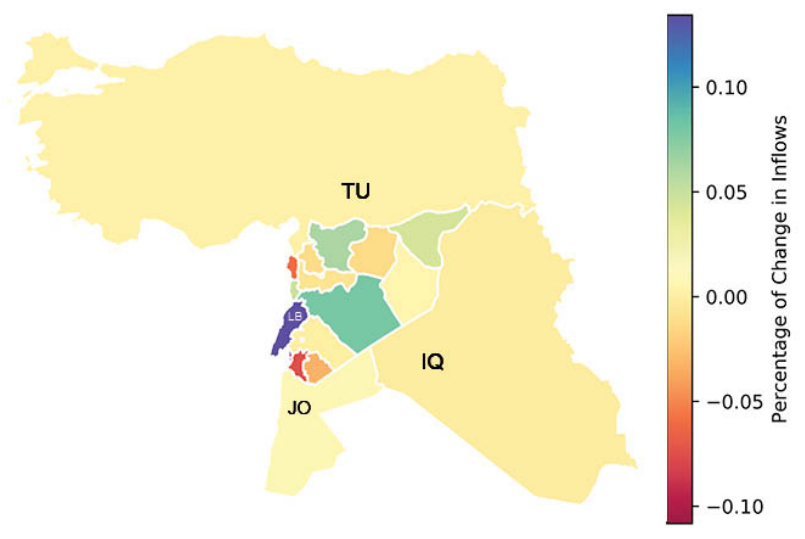

Figure 5. Change of inflow towards possible destinations as a result of Iraqi border closure. The colors reveal the difference between the outcomes from the control experiment and the Iraqi border closure experiment averaged over 10 months. Once the journey towards Iraq is impossible, Lebanon as a neighboring country becomes a destination for many more refugees than before.

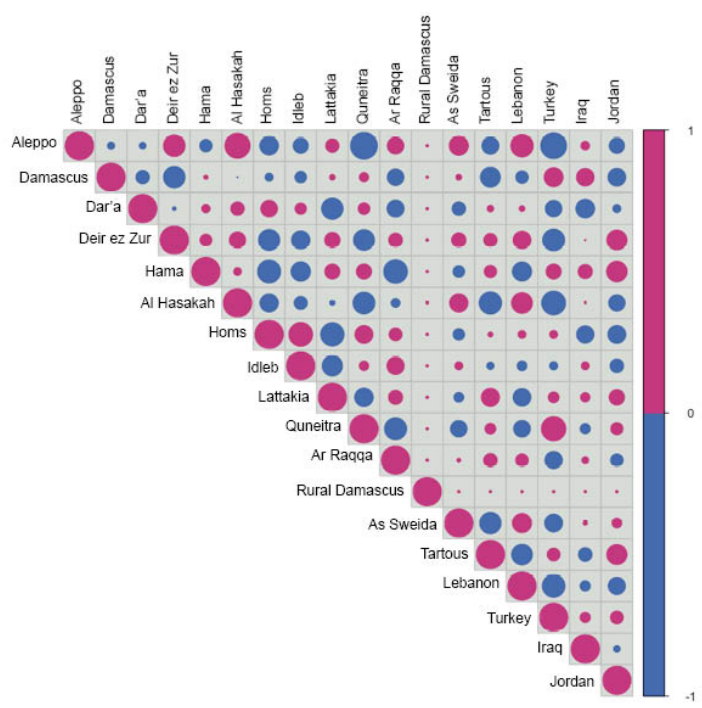

Figure 4. Correlation diagram of change in inflow towards possible destinations as a result of Turkish border closure. Each cell represents the correlation over time between the changes resulting from the Turkish border closure in the two regions. A positive correlation, for example, means that increased (decreased) flow to one location was associated with increased (decreased) flow to the other. The size of circle reflects the magnitude of correlation.

This experiment demonstrates how not all the border closures have the same effect. In this case, in contrast with the previous experiment, a large increase in journeys towards international borders emerges. This is particularly true for the case of Lebanon, which experiences a higher inflow comparing to the Syrian governorates (on average, 300 individuals). On average, Iraqi border closure led to 365 more refugees overall, whereas the Turkish border closure decreased the total legal border crossings by 6660 individuals during the 10 month period. As in the previous case, the correlogram of displacements is shown in Figure 6. Again, substantive associations can be seen between locations which are not direct neighbours of each other. At a higher level, comparison of the two correlograms (Figures 4 and 6) shows that the effects differ between the two cases. This again highlights the complexity of the system: the coupling between locations is not simply a function of their spatial arrangements, but depends on context.

Figure 7 further illustrates our result by showing the volume of refugees produced under the different experimental scenarios. While the Turkish border closure results in a large net decrease, the scenario relating to Iraq in fact results in a small increase. This further illustrates the non-equivalence of the two borders: the population that travels to Iraq (when the border is open) is fundamentally different to that which travels to Turkey, and they exhibit different preferences with the respective options are removed.

\section{DISCUSSION}

Motivated by the increasingly complex refugee crisis all across the globe, the aim of this paper has been to demonstrate the strength and applicability of agent-based modelling combined with spatio-temporal data in understanding the role and consequences of policy intervention in IDP and refugee movements. Incorporating data and designing a data-driven framework in order to analyse the effect of policy intervention is a relatively new approach in migration studies. The research presented here seeks insight into the behaviour of individuals during an episode of conflict in Syria under border-related policy intervention. The underlying behaviour of migrants often involves a decision to leave and a choice of destination, both of which are influenced by the spatio-temporal dynamics of

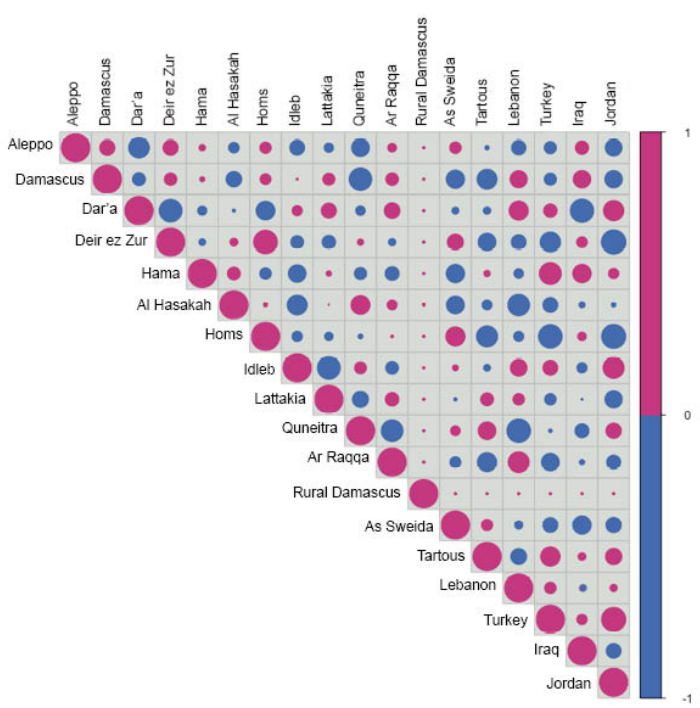

Figure 6. Correlation diagram of change in inflow towards possible destinations as a result of Iraqi border closure. The diagram shows how a decrease in inflows towards Iraq is correlated with increase in Lebanon and Jordan. 
environmental factors at their current location and potential destinations.

Having the aim to study the effect of policy intervention in mind, we have developed a data-driven agent-based model to establish a basis for intended scenario experiments. The model is not only able to replicate the general features of the flow pattern, but also provides a quantitatively close fit to the empirical data. Whilst simple, the model incorporates important cues and fundamental features of conflict-induced displacement both in its structure and empirically. We have used the model to explore how two cases of policy intervention - Turkish border closure and Iraqi border closure, both of which are major border control policies available to be enacted by the governments - might affect the final pattern of displacement within Syria and across the neighbouring countries. Such exploration has clear implications for policy. To capture the effect of such interventions, we quantified border functionality and incorporated both empirical data and synthetic scenarios to simulated experiments.

A fundamental output from the introduced model is the identification of the main destinations which would be receiving higher number of refugees as a result of each border closure scenario, as well as the magnitude of change for each destination. Furthermore, the experiments are found to reveal different emergent patterns as a result of enacted closure at different borders in the spatial network of Syria.

Both closures result in inflows being displaced towards other destinations, both within and outside Syria. However, Iraqi border closure revealed a different pattern comparing to Turkish border closure. This suggests an underlying difference between the two scenarios, and suggests that the two populations (those who migrate to Turkey or Iraq in the non-closure scenario) are distinct in their actions and motivation. One explanation relates to their different spatial location.

The Turkish border, which has the highest number of crossing points, while bordering a number of highly conflicted areas, represents the only realistic (legal) option for international migration for many individuals in the northern region. Its closure therefore removes the possibility of international migration, and so realistically individuals either manage to take refuge internally within Syria (as IDPs), or they decide to take illegal pathways towards an international border. The geospatial features of the Iraqi border, on the other hand, gave rise to higher number of border crossings towards Lebanon as opposed to the case of Turkish border closure. The migrants who would otherwise cross into Iraq have access to a suitable alternative, to which they can travel.

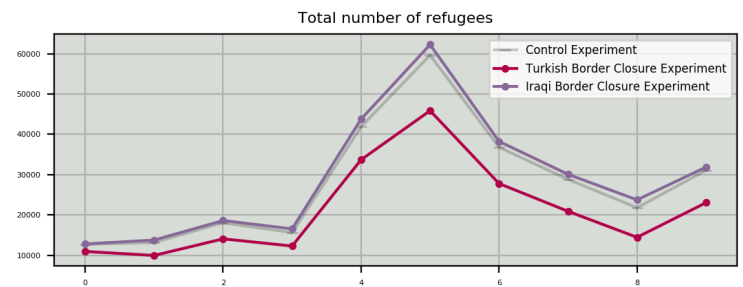

Figure 7. Total number of refugees per month. As opposed to Turkish border closure, Iraqi border closure resulted in more refugees comparing to the control experiment.
Of interest for future work is the question of whether similar patterns are observed for borders further removed from Syria (e.g. in Europe). While border closures further afield are often proposed, their 'net' effect - particularly whether they reduce migration or simply displace it (and, if so, to where) is poorly understood. An extension of this model may be used to explore such questions.

Given the spatial configuration of Syria, the model resulted in a variety of outcomes that inform proposed policy interventions. The model extensively uncovered the impacts of such interventions. There are a range of scenarios to be explored using the model - such as camp placement strategies and aid distribution - that are beyond the scope of this paper. The model has the potential to incorporate more variables and highly granular datasets in order to analyse a range of policies and produce realistic patterns. More sophisticated decision models can be incorporated with the ultimate goal of generating further quantitative evidence relating to more policy interventions. Among further developments for our future work, the incorporation of social network and behavioural heterogeneity will substantially enhance the performance and applicability of the discussed framework to analyse refugee-related policies.

\section{References}

Carlson, M., Jakli, L., and Linos, K. (2018). Rumors and refugees: How government-created information vacuums undermine effective crisis management. International Studies Quarterly, 62(3):671-685.

Charlson, F., van Ommeren, M., Flaxman, A., Cornett, J., Whiteford, H., and Saxena, S. (2019). New WHO prevalence estimates of mental disorders in conflict settings: a systematic review and meta-analysis. The Lancet, 394(10194):240-248.

Chu, T., Ghosn, F., Simon, M., Braithwaite, A., and Frith, M. (2019). The journey home: Flight related factors on refugee decisions to return. Americal Political Science Association.

Crawley, H., Duvell, F., Jones, K., Mchmahon, S., and Sigona, N. (2017). Unravelling Europe's 'migration crisis'. Bristol University Press.

Frydenlund, E., Foytik, P., Padilla, J. J., and Ouattara, A. (2018). Where are they headed next? Modeling emergent displaced camps in the DRC using agent-based models. In 2018 Winter Simulation Conference (WSC), pages 22-32. ISSN: $1558-4305$.

Frydenlund, E., Jones, E. C., and Padilla, J. J. (2019). Mobility in crisis: An agent-based model of refugees' flight to safety. In New Approaches to the Scientific Study of Religion, pages 191-208. Springer International Publishing.

Gigerenzer, G. and Gaissmaier, W. (2011). Heuristic decision making. Annual Review of Psychology, 62(1):451-482.

Haas, H., Czaika, M., Flahaux, M.-L., Mahendra, E., Natter, K., Vezzoli, S., and Villares-Varela, M. (2019). International migration: Trends, determinants, and policy effects. Population and Development Review, 45(4):885-922. 
Hébert, G. A., Perez, L., and Harati, S. (2017). An agent-based model to identify migration pathways of refugees: The case of syria. In Advances in Geographic Information Science, pages 45-58. Springer International Publishing.

Heppenstall, A. J., Evans, A. J., and Birkin, M. H. (2007). Genetic algorithm optimisation of an agent-based model for simulating a retail market. Environment and Planning B: Planning and Design, 34(6):1051-1070.

Klabunde, A. and Willekens, F. (2016). Decision-making in agent-based models of migration: State of the art and challenges. European Journal of Population, 32(1):73-97.

Kniveton, D. R., Smith, C. D., and Black, R. (2012). Emerging migration flows in a changing climate in dryland Africa. Nature Climate Change, 2(6):444-447.

Massey, D. S., Arango, J., Hugo, G., Kouaouci, A., Pellegrino, A., and Taylor, J. E. (1993). Theories of International
Migration: A Review and Appraisal. Population and Development Review, 19(3):431-466. Publisher: [Population Council, Wiley].

Mohammadi, A., Asadi, H., Mohamed, S., Nelson, K., and Nahavandi, S. (2017). Openga, a c++ genetic algorithm library. In Systems, Man, and Cybernetics (SMC), 2017 IEEE International Conference on, pages 2051-2056. IEEE.

Simon, M. (2019). Path dependency and adaptation: The effects of policy on migration systems. Journal of Artificial Societies and Social Simulation, 22(2).

Simon, M., Schwartz, C., Hudson, D., and Johnson, S. D. (2018). A data-driven computational model on the effects of immigration policies. Proceedings of the National Academy of Sciences, 115(34):E7914-E7923.

Suleimenova, D., Bell, D., and Groen, D. (2017). A generalized simulation development approach for predicting refugee destinations. Scientific Reports, 7(1). 